\title{
Atomic Resolution Study of Local Strains in Doped $\mathrm{VO}_{2}$ Nanowires
}

\author{
Hasti Asayesh-Ardakani ${ }^{1,2}$, Anmin Nie ${ }^{1,2}$, Peter M. Marley ${ }^{3}$, Sujay Singh ${ }^{4}$, Patrick J. Philips ${ }^{2}$, \\ Farzad Mashayek ${ }^{5}$, Ganapathy Sambandamurthy ${ }^{4}$, Ke-bin Low ${ }^{6}$, Robert F. Klie ${ }^{2}$, \\ Sarbajit Banerjee $^{3}$, Gregory M. Odegard ${ }^{1}$ and Reza Shahbazian-Yassar ${ }^{1,2,5}$ \\ 1. Department of Mechanical Engineering, Michigan Technological University, Houghton, MI $49933-$ \\ 1295, USA \\ 2. Department of Physics, University of Illinois at Chicago, Chicago, IL60607-7059, USA \\ ${ }^{3 .}$ Department of Chemistry, University at Buffalo, State University of New York, Buffalo, New York \\ 14260-3000, USA \\ 4. Department of Physics, University at Buffalo, State University of New York, Buffalo, New York \\ 14260-3000, USA \\ 5. Department of Mechanical and Industrial Engineering, University of Illinois at Chicago, Chicago, \\ IL60607-7059, USA \\ 6. Research Resource Center, University of Illinois at Chicago, IL60607-7059, USA
}

Vanadium dioxide $\left(\mathrm{VO}_{2}\right)$, one the correlated electron material has received many attentions through a metal-insulator transition (MIT) at $\sim 340 \mathrm{~K}$, close to room temperature [1]. The MIT in $\mathrm{VO}_{2}$ is associated with a structural phase transition, sharp resistivity and optical transparency changes by several order of magnitudes [2]. The phase transition occurs from a monoclinic (M) structure to a tetragonal rutile $(\mathrm{R})$ structure. The unique properties of $\mathrm{MIT}$ in $\mathrm{VO}_{2}$ have proposed many applications such as thermo/electrochromics, Mott transistors, memristors, thermal actuators, gas sensors, strain sensors and temperature sensors.

The present work investigates the effect of $\mathrm{W}$ dopants in MIT of individual single-crystalline $\mathrm{VO}_{2}$ nanowires by use of aberration corrected scanning transition electron microscopy and in situ TEM. $\mathrm{W}_{x} \mathrm{~V}_{1-x} \mathrm{O}_{2}$ nanowires with different doping concentration of $\mathrm{W}(0.2$ and 0.8 at.\%) were used in this study which correspond to transition temperatures of $330 \mathrm{~K}$ and $290 \mathrm{~K}$ for samples with 0.2 at. $\%$ and 0.8 at. $\%$ $\mathrm{W}$, respectively as reported by $\mathrm{Wu}$ et al. [3].

The atomic scale Z-contrast imaging of individual single-crystalline $\mathrm{W}_{x} \mathrm{~V}_{1-x} \mathrm{O}_{2}$ nanowires indicates $\mathrm{W}$ dopant atoms in the structure as shown in Figure 1a. Geometric phase analysis (GPA) of high-resolution images reveals the effect of dopants in MIT of $\mathrm{VO}_{2}$ (Figure 1b-c). W dopants introduce structural distortions on the $\mathrm{VO}_{2}$ structure. The comparison between induced distortions in the structure of 0.2 and 0.8 at. $\% \mathrm{~W}$ nanowires reveal that the $\mathrm{W}$ dopants induce a greater stress in the normal direction of $(\overline{1} \overline{2} 2)$ lattice planes of monoclinic structure and this stress facilitates the phase transition form monoclinic structure to tetragonal structure. We also verified the experimental observation of by Density Functional Theory (DFT) calculations. The second part of this study, the MIT is investigated by electrical testing inside the chamber of a TEM. The in situ electrical characterization illustrates insulating monoclinic structure for low doped nanowires and metallic tetragonal structure for highly doped nanowires at room temperature. 


\section{References:}

[1] FJ Morin, Phys Rev Lett 3 (1959), p. 34.

[2] V Eyert, Ann Phys (Berlin) 11 (2002), p. 650.

[3] T-L Wu, Whittaker L, Banerjee S and Sambandamurthy G, Phys Rev B 83 (2011), p. 073101.
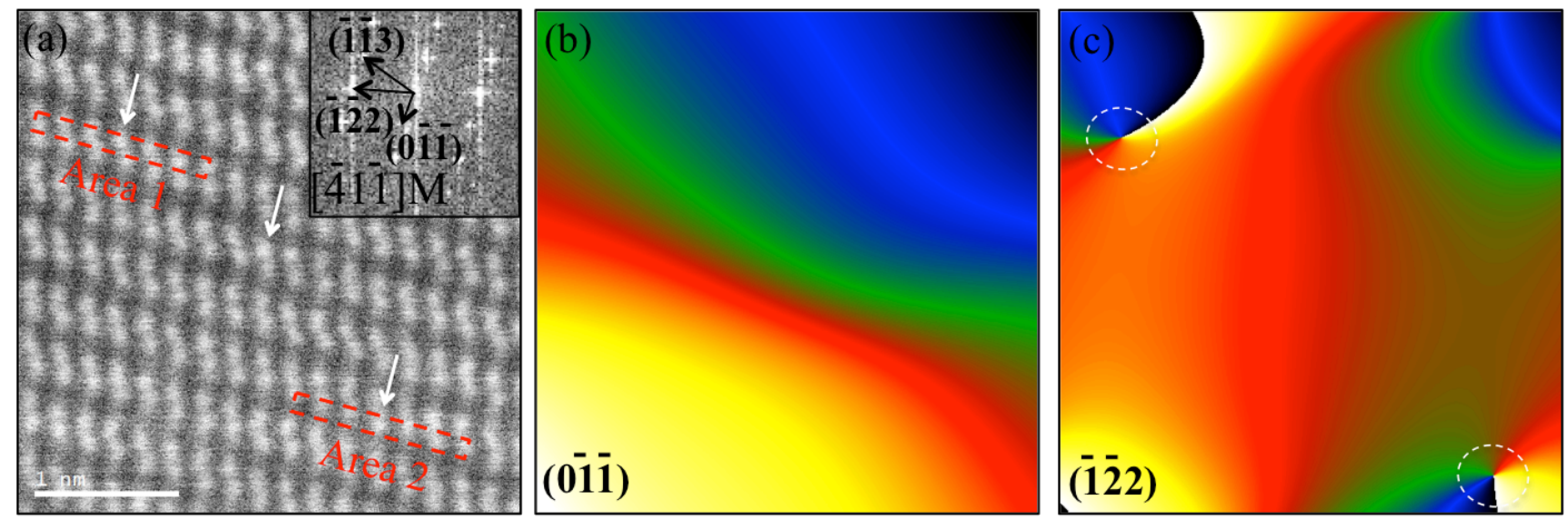

Figure 1. (a) Atomic resolution HAADF image of $\mathrm{W}_{x} \mathrm{~V}_{1-x} \mathrm{O}_{2}$ nanowires $(x=0.2$ at. \%). Insets correspond to the FFT of (a) which indicates that (a) has been acquired along the [ $\overline{4} 1 \overline{1}]$ zone axis of the monoclinic structure. The spots with higher intesity implie the existance of $\mathrm{W}$ in each column as compared to other spots. (b-c) The geometric phase images relate to $(0 \overline{1} \overline{1})$ and ( $\overline{1} \overline{2} 2$ ) lattice planes. (c) shows lattice distortions in (122) lattice planes of monoclinic structure. 